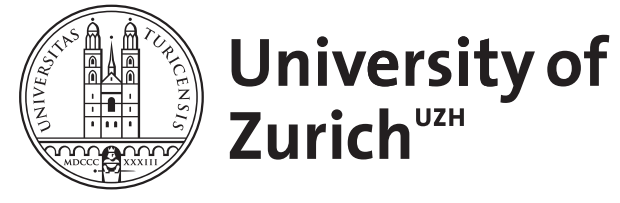

\title{
Unraveling the Interplay of Community Assembly Processes Acting on Multiple Niche Axes across Spatial Scales
}

Trisos, Christopher H ; Petchey, Owen L ; Tobias, Joseph A

DOI: https://doi.org/10.1086/678233

Posted at the Zurich Open Repository and Archive, University of Zurich ZORA URL: https://doi.org/10.5167/uzh-102287

Journal Article

Published Version

Originally published at:

Trisos, Christopher H; Petchey, Owen L; Tobias, Joseph A (2014). Unraveling the Interplay of Community Assembly Processes Acting on Multiple Niche Axes across Spatial Scales. The American Naturalist, 184(5):593-608.

DOI: https://doi.org/10.1086/678233 


\title{
Unraveling the Interplay of Community Assembly Processes Acting on Multiple Niche Axes across Spatial Scales
}

\author{
Christopher H. Trisos, ${ }^{1,2, \star}$ Owen L. Petchey, ${ }^{3}$ and Joseph A. Tobias ${ }^{1}$
}

1. Edward Grey Institute, Department of Zoology, University of Oxford, Oxford OX1 3PS, United Kingdom; 2. Fynbos Node, South African Environmental Observation Network, Private Bag X7, Rhodes Drive, Newlands 7735, South Africa; 3. Institute of Evolutionary Biology and Environmental Studies, University of Zurich, CH-8057 Zurich, Switzerland

Submitted August 22, 2013; Accepted June 18, 2014; Electronically published October 1, 2014

Online enhancements: appendixes, zip file. Dryad data: http://dx.doi.org/10.5061/dryad.k9m20.

\begin{abstract}
How the relative importance of community assembly processes varies with spatial scale is the focus of intensive debate, in part because inferring the scales at which specific niche-based processes act is difficult. One obstacle is that standard phylogenetic and functional diversity metrics may integrate the signals of multiple processes when combining separate niche axes into one variable (multiple-niche-axis metrics), potentially obscuring overlapping niche-based processes. We use simulations to evaluate the power of these metrics to detect competition and habitat filtering when these processes operate across multiple niche axes and vary in their relative importance. We then test for both processes at a range of spatial scales in a Neotropical bird assemblage. Simulations revealed that multiple-niche-axis metrics had low power to detect competition and habitat filtering when a mix of both processes acts across niche axes, whereas metrics focused on singleniche axes were better able to deal with this complexity. We found the same contrast in bird communities, where both competition and habitat filtering were detected at the scale of individual territories, but only by single-niche-axis metrics focused on specific niche axes (e.g., foraging traits). Our results suggest that multiple-niche-axis metrics may produce misleading evidence that niche-based processes are partitioned, particularly across scales, and highlight the importance of analyzing functional diversity patterns on individual niche axes when testing assembly models.
\end{abstract}

Keywords: community assembly, environmental filtering, interspecific competition, niche differentiation, phylogenetic community structure, Neotropical birds.

\section{Introduction}

The study of differences in species resource-use strategies and their implications for species coexistence has dominated ecology throughout much of the last century, with evidence for ecological niche partitioning discovered in communities as diverse as yeast cell cultures, plants, and birds (Gause 1934; Stubbs and Wilson 2004; Lovette and

\footnotetext{
* Corresponding author; e-mail: trisos@saeon.ac.za.

Am. Nat. 2014. Vol. 184, pp. 000-000. (C) 2014 by The University of Chicago. 0003-0147/2014/18405-54917\$15.00. All rights reserved. DOI: $10.1086 / 678233$
}

Hochachka 2006). Most recent attention has focused on clarifying the importance of niche-based processes in structuring communities relative to purely neutral models (Hubbell 2001; Chase and Myers 2011), a debate that has led to a more inclusive concept of the processes involved in community assembly, including niche-based habitat filtering, interspecific competition, dispersal, and demographic stochasticity (Vellend 2010; Weiher et al. 2011). However, this expanded conceptual framework is rarely specific with regard to the spatial scales at which different processes have the greatest influence, and the potential interaction of these processes across scales remains unclear (Levin 1992; McGill 2010; Chase and Myers 2011; Chave 2013).

Ascertaining the grain sizes (the scales of the local community or sample plot) at which niche-based processes operate is critical for understanding how scale dependency in these processes influences species co-occurrence (Huston 1999; Weiher et al. 2011). It is generally assumed that interspecific competition is strongest at the scale of direct interactions among individuals and therefore becomes progressively weaker as grain size increases (Weiher and Keddy 1995; Vamosi et al. 2009). In contrast, habitat features such as topography and soil type typically vary over greater distances than interactions between individuals, such that habitat filtering is expected to be strongest at grain sizes larger than those for competition (Swenson et al. 2007; Kraft and Ackerly 2010) but small enough to exclude habitat heterogeneity (Weiher et al. 2011).

The classic signature of this transition in niche-based processes across grain sizes is a niche-differentiated pattern of regular spacing or overdispersion in the trait values of co-occurring species at small grain sizes (MacArthur and Levins 1967; Ricklefs and Travis 1980), shifting to the opposite pattern of clustering of species' trait values within communities at intermediate grain sizes, especially when spread across large spatial extents that encompass increased habitat heterogeneity (Weiher and Keddy 1995; 
Swenson et al. 2007). Similarly, to the extent that closely related species share similar trait values, community phylogenetic structure should shift from the co-occurrence of more distantly related (phylogenetic overdispersion) to more closely related (phylogenetic clustering) species than expected by chance with increasing spatial scale (Swenson et al. 2007; Cavender-Bares et al. 2009). Yet, the generality of this transition across scales is poorly understood because previous studies have focused on only a few taxonomic groups and produced mixed results (Vamosi et al. 2009; McGill 2010).

Most analyses exploring community assembly processes across scales have focused either on plants or microbes (e.g., Cadotte 2006; Swenson and Enquist 2009; Kraft and Ackerly 2010), as these organisms are highly amenable to observational and experimental tests. In contrast, it is relatively difficult to define communities and delimit sampling scales in mobile animal species, particularly vertebrates (Vamosi et al. 2009). Because of this long-standing research bias, our knowledge of the ecological and evolutionary patterns describing communities is primarily based on systems where the spatial scale of interactions between individuals is likely to be highly circumscribed, perhaps underestimating the signature of competition at larger scales (e.g., Gotelli et al. 2010). Alternatively, high mobility may allow species to mediate competitive interactions rapidly and thus result in communities that appear randomly assembled or even clustered at smaller spatial scales (Gómez et al. 2010; Weiher et al. 2011; HarmonThreatt and Ackerly 2013).

Another important source of uncertainty relates to analytical techniques. Most recent studies of community assembly across scales have applied phylogenetic or traitbased metrics that are sensitive to both overdispersion and clustering (e.g., Cavender-Bares et al. 2006). Moreover, many of these trait-based metrics combine multiple traits into a single analysis (e.g., functional diversity [FD]; Petchey and Gaston 2002) or do so indirectly in the case of phylogenetic methods (Webb et al. 2002). Because different traits are often associated with different niche axes (Violle et al. 2007), metrics that combine traits from multiple niche axes may have the advantage of providing an integrated overview of community structure. However, when different assembly processes act on separate niche axes independently or exert combined effects on the same niche axis, a potential drawback is that these multipleniche-axis metrics may combine the signals of contrasting assembly processes (Swenson and Enquist 2009). Such metrics may therefore obscure the niche-based assembly processes involved at a given scale if one process masks another or if multiple processes cancel each other's signal, generating patterns consistent with neutral dynamics (Kraft et al. 2007; Weiher et al. 2011). For example, Spa- sojevic and Suding (2012) found that along a plant productivity gradient, multitrait FD did not differ from FD expected under random assembly because functional diversity in traits associated with above- and belowground competition showed significant but opposite patterns with increasing resource availability.

Despite the relevance of this issue for improving the accuracy of inferences about community assembly (see Aiba et al. 2013), it is not yet known how most multipleniche-axis metrics of community phylogenetic or trait structure vary in their sensitivity to a given niche-based assembly process when multiple processes do not all act on the same niche axes and particularly when different processes vary in their relative strength. Also largely unknown is whether single-niche-axis metrics increase rates of detection of niche-based processes, thereby revealing different patterns across spatial scales. Addressing these methodological issues is essential in order to connect the results of analyses using functional and phylogenetic diversity metrics to community assembly processes.

This article has two aims: (1) to evaluate the power of multiple-niche-axis (including phylogenetic) and singleniche-axis metrics to detect interspecific competition and habitat filtering when these processes operate across multiple niche axes and vary in their relative strength, and (2) to test for the predicted shift in niche-based assembly processes from interspecific competition to habitat filtering in Neotropical bird communities across four nested grain sizes (0.8-6.4 ha) using multiple-niche-axis and singleniche-axis metrics. Tropical insectivorous birds provide an excellent case study of scale dependency in vertebrate community assembly. They can be efficiently surveyed to generate point communities-that is, assemblages of species with high likelihood of interaction because their home ranges overlap at a single point. In addition, many species hold territories year-round and defend them against heterospecifics (Robinson and Terborgh 1995), suggesting that competitive exclusion may extend considerably further than the size of individual organisms. Our assessment of metric performance (aim 1) was based primarily on simulated communities, but the size of the species pool, local species richness, and trait data for community simulations were obtained from observed bird community and trait data (aim 2).

\section{Methods \\ Neotropical Bird Community Data}

We conducted bird surveys from July through November 2010 in the Kosñipata Valley at Wayqecha Biological Research Station, Peru (lat. $13^{\circ} 10^{\prime} 35^{\prime \prime} \mathrm{S}$, long. $71^{\circ} 35^{\prime} 20^{\prime \prime} \mathrm{W}$ ), at 72 survey points spaced at regular $120-\mathrm{m}$ intervals on a 
grid layout (fig. 1). The grid covered an elevation range of 2,570-3,050 $\mathrm{m}$ and contained two major habitat typesmontane cloud forest and high-elevation puna grasslandin roughly equal proportion (figs. 1, A1; figs. A1-A6 are available online). A third shrub habitat type was present in areas where the ecotone between the cloud forest and grassland was wider. For a full description of vegetation types and topography across the site, see Gibbon et al. (2010).

At each of the 72 points, we conducted standardized audial and visual bird surveys, focused on circular areas with a $50-\mathrm{m}$ radius centered on each point, ensuring that neighboring survey-point communities were nonoverlapping. The grain size of individual survey-point communities was approximately 0.8 ha. The total extent of our study site is small enough ( $~ 80$ ha) that most study species are able to disperse across the site within minutes, and even the most nondispersive species (e.g., Scytalopus) are potentially able to do so within a single generation. See appendix A (apps. A, B are available online) for a full description of survey methods.

Forty-one different species were observed across all 72 points (table A1; tables A1-A8 are available online). This observed species richness represented $91 \%$ of the estimated true species richness for the study site (Chao II incidencebased estimator [Chao 1987] implemented in EstimateS [Colwell 2000]). In addition, the mean proportion of new species detected at a survey point leveled off at $<0.05$ species per visit after five visits (fig. A2). Thus, our surveys captured a high proportion of bird diversity across the study site.

Community survey, vegetation survey, and functional trait data for all species are available online in the Dryad Digital Repository: http://doi.org/10.5061/dryad.k9m20 (Trisos et al. 2014).

\section{Trait Data and Associated Niche Axes}

As most community assembly models apply to interactions within trophic levels (Hubbell 2001; Cavender-Bares et al. 2009), we restricted our analyses to bird species with primarily insectivorous diets. The link between morphological traits and ecology is relatively well established in birds, as the avian beak is a classic index of trophic niche (Hutchinson 1959; Schoener 1965; Grant and Grant 2006) and other biometric measurements such as tarsus and wing length can be related to foraging maneuver, microhabitat, and substrate use (Miles and Ricklefs 1984), helping us to identify candidate sets of traits that represent key niche axes potentially important in community assembly. We measured six functional traits (beak length, beak width, beak depth, wing length, tail length, and tarsus length) from 1 to 70 (mean \pm SE: $11.54 \pm 2.43$ ) individuals. Data for most species were sampled from individuals captured by mist-netting at the study site, with missing trait values for seven species measured on museum specimens from localities as close as possible to the study site. Measurements of live birds and museum specimens were taken

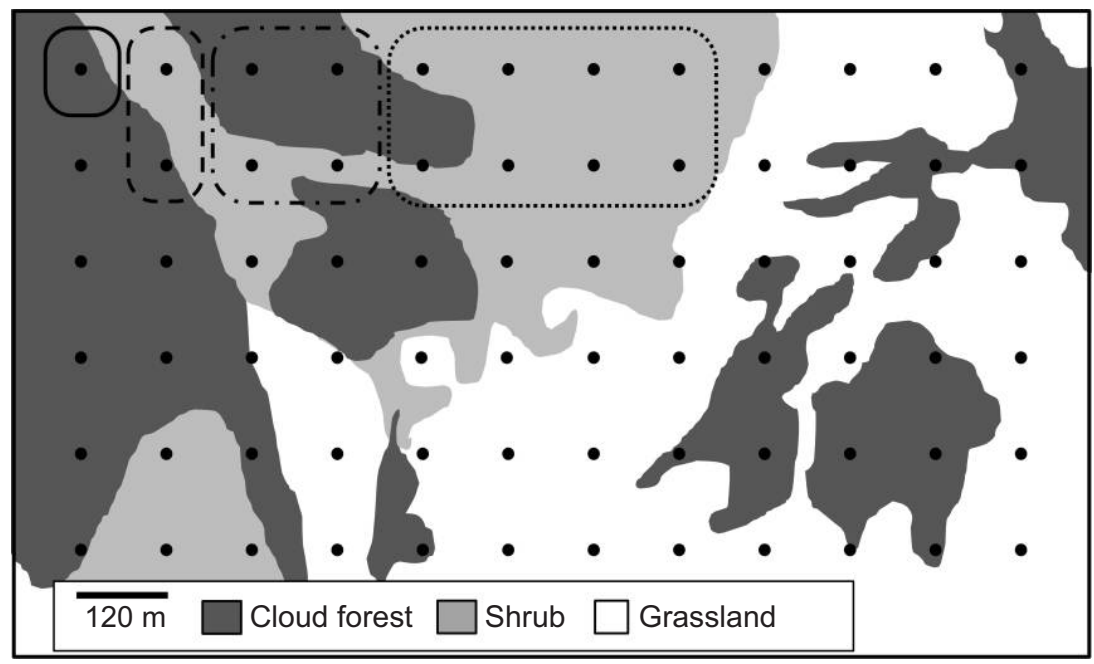

Figure 1: Diagram of the study grid. Outlined areas (top left) indicate how individual survey-point communities were combined with neighboring points in the study area to create communities at four nested spatial scales (grain sizes): 0.8 ha (solid line), 1.6 ha (dashed line), 3.2 ha (dash-dotted line), and 6.4 ha (dotted line). Sample size was 72 communities for the smallest grain size and 9 communities for the largest. The relationship between points and habitats is shown in grayscale; see figure A1 for a satellite image. 
using standard procedures described in appendix A. Because of the difficulty of sampling a larger number of birds using mist-netting, and because the proportion of variance (calculated using ANOVA) in trait values explained by species $(84.5 \%-94.7 \%)$ was far greater than that explained by intraspecific differences $(5.3 \%-15.5 \%)$ for the 21 bettersampled species ( $n>5$ individuals; table A2), we used mean trait values normalized with log transformations for all analyses, following previous trait-based studies (Stubbs and Wilson 2004; Kraft et al. 2008).

Trait-based tests for niche-based assembly processes require knowledge of relevant niche axes and traits. For example, if foraging differences are accentuated among coexisting species to reduce interspecific competition, it seems sensible to focus tests for the signature of competitive exclusion (i.e., overdispersion) on key trophic traits such as beak shape. However, the link between traits and niche axes is not always so clear, and sets of traits often provide information about the same or overlapping niche axes. In our data set, functional traits were strongly positively correlated $(r=0.28-0.84)$, largely through their association with overall body size. To prevent these correlations from biasing analyses toward detecting only processes associated with body size, we used ordination techniques (based on a two-step principal component analysis [PCA]) to derive independent trait axes (hereafter termed derived trait axes; for details, see "Results"; fig. A3; app. A).

\section{Multiple-Niche-Axis Metrics}

We used two standard metrics, FD (Petchey and Gaston 2002) and convex hull volume (CHV; Cornwell et al. 2006), both of which test concurrently for habitat filtering and niche differentiation. The metrics FD and CHV are generally applied to sets of multiple functional traits and, because they test for two patterns concurrently, can be classified as multipattern, multiple-niche-axis metrics. Functional diversity is a measure of how dispersed a set of species is in trait space (Petchey and Gaston 2002), while convex hull volume is the smallest convex set in trait space enclosing all of the species trait values within a community and is analogous to a multivariate measure of the range of community trait values (Cornwell et al. 2006). We selected FD and CHV because they are widely used with presence/absence data and have been shown to have high power to detect trait clustering (CHV) and overdispersion (FD) in previous community assembly simulations (Mouchet et al. 2010; Aiba et al. 2013).

We calculated FD and CHV using all three derived trait axes. These trait axes were standardized to have a mean of zero and unit variance, given the absence of any a priori weighting on the ecological importance of the axes (Vil- léger et al. 2008). Functional diversity measures for each community were standardized by the FD of the total species pool so that variation in FD ranged from 0 to 1 . For both FD and CHV, results using unstandardized trait axes were very similar to those reported here (table A8).

Another source of multipattern, multiple-niche-axis metrics that are now widespread in community ecology are phylogenetic analyses (Cavender-Bares et al. 2009). A standard assumption of community phylogenetic models is that multiple niches are phylogenetically conserved and, thus, that co-occurring species should be more related than expected by chance under habitat filtering but less related under competitive exclusion (Kraft et al. 2007; but see Mayfield and Levine 2010). To test this, we constructed a molecular phylogeny for all species in our study community (fig. 3; see app. A for all information on genetic extraction, sequencing, and tree-building methodology). We assessed the phylogenetic signal in functional traits using Blomberg's $K$ statistic (Blomberg et al. 2003) and in habitat type using $D$ (Fritz and Purvis 2012), both with 1,000 permutations. We selected the metrics mean phylogenetic distance (MPD) and mean nearest taxon distance (MNTD) because they are two of the most commonly used measures of community relatedness and have been the subject of previous power analyses (Kraft et al. 2007). Mean phylogenetic distance is the mean of the pairwise phylogenetic distances between co-occurring species and is most sensitive to tree-wide patterns of phylogenetic clustering and evenness. Mean nearest taxon distance is the mean of the phylogenetic distances separating each species from its closest co-occurring relative and is most sensitive to patterns of phylogenetic clustering or evenness at the tips of the phylogeny (Webb et al. 2002; Kraft et al. 2007).

\section{Single-Niche-Axis Metrics}

We analyzed each derived trait axis individually using metrics we term either multipattern, single-niche axis, because they test for both assembly patterns (clustering and overdispersion), or single-pattern, single-niche axis, because they test for only one assembly pattern. We focused on three multipattern, single-niche-axis metrics: (1) FD applied to a single trait axis, (2) variance (the variance in species values within a community along a single trait axis), and (3) range (the range in species trait values within a community, i.e., the single-niche-axis equivalent of $\mathrm{CHV}$ ). Both variance and range have previously been used predominantly as a measure of trait clustering (e.g., Stubbs and Wilson 2004; Kraft and Ackerly 2010). We also focused on one single-pattern, single-niche-axis metric, SDNDr, the standard deviation of the distances between neighboring species along a single trait axis, divided by the trait range of the community. The metric SDNDr is used to 
detect only the regular spacing in species trait values predicted by competitive exclusion (Kraft and Ackerly 2010).

\section{Community Assembly Simulations and Power Analyses}

Community assembly simulations were used to assess the power of multiple-niche-axis and single-niche-axis metrics to detect competitive exclusion (based on limiting similarity) and habitat filtering (based on trait clustering), operating over multiple niche axes and across a range in the relative importance of each process. We assembled 1,000 communities of nine species (the median species richness for an individual survey-point community in our data set) for each of the five scenarios of relative importance of habitat filtering and competitive exclusion, varying from the sole action of one process to a $50: 50 \mathrm{mix}$ in the importance of both processes. We assembled communities using the three uncorrelated derived trait axes generated by the two-step PCA (see app. A; fig. A3). The body-size trait axis was subjected to habitat filtering and limiting similarity, the locomotory trait axis (tarsus to tail length ratio) to habitat filtering only, and the trophic trait axis (beak shape) to limiting similarity only. We also performed simulations where the axes were switched so that the locomotory trait axis was subjected to habitat filtering and limiting similarity and the body-size axis to habitat filtering only. This was done to investigate whether the distribution of a trait's values in the species pool had an effect on the power of the metrics.

Simulated communities were assembled by removing species from the pool of 41 species recorded at the study site, using the algorithms of Kraft et al. (2007). First, the habitat-filtering algorithm selected an optimal trait value at random from within the observed range of each of the traits exposed to habitat filtering; a number of species, set by the relative importance of habitat filtering, furthest from this optimum combination of trait values-by $\mathrm{Eu}$ clidean distance-were removed. Subsequently, the limiting similarity algorithm detected the pair of species with the smallest Euclidean distance between them along the trait axes exposed to competition and removed one of the pair at random, with this process repeated until the desired local community species richness was reached. To vary the relative importance of habitat filtering and competitive exclusion, the number of species eliminated due to either process was adjusted, with the $50: 50$ assembly scenario resulting in an equal number (16) being eliminated by each process. To enable calculation of the phylogenetic metrics, we constructed a community phylogeny for each simulated community by selecting, from the phylogeny for all species at the study site, the nine species in each simulated community.

The observed value and the null model mean and stan- dard deviation from 999 null communities were calculated for each multiple-niche-axis and single-niche-axis metric for each simulated community. The null model drew species at random from the species pool while maintaining the species richness for each community. It was not necessary to use a null model that maintained species occurrence frequency because there was no species occurrence frequency structure in the simulated communities independent of that generated by the assembly algorithms we applied. To test the power of the metrics to reject a false null hypothesis of random assembly, we sampled (without replacement) sets of 20 communities from the 1,000 communities simulated for a given scenario of habitat filtering to competitive exclusion. This procedure yielded 50 sets of local communities for each of the five assembly scenarios. Wilcoxon signed-rank tests were then performed on each of these 50 sets of 20 local communities, allowing the proportion of tests rejecting the null model for a given scenario to be recorded. The Wilcoxon signed-rank test assessed whether the distribution of the differences between the observed, simulated local community values and their respective null model means was shifted away from zero. For all multipattern metrics, a two-tailed test was used, with a shift in observed values below zero indicative of clustering and a shift above zero of overdispersion (Kraft et al. 2008; Kraft and Ackerly 2010). Following previous studies (e.g., Kraft et al. 2008; Kraft and Ackerly 2010), a one-tailed test for a shift below zero was used for SDNDr because this single-pattern metric is designed to test only for reduced standard deviation in neighbor distances (regular spacing). We note that using Wilcoxon signed-rank tests to evaluate the power of the metrics at the aggregate level of multiple individual communities is likely to yield higher estimates of power than that obtained when testing individually for the deviation of single communities from the null model (Kraft and Ackerly 2010). All simulations and metric analyses were performed in R (R Core Team 2013). See appendix A for further details of analytical techniques and $\mathrm{R}$ code.

\section{Tests of Bird Community Assembly across Scales}

To investigate variation in habitat filtering and interspecific competition across grain sizes, we combined communities of the smallest grain size ( 0.8 ha) with neighboring communities in pairs, quadruples, and eights to generate communities at larger scales (1.6, 3.2, and 6.4 ha; fig. 1), while keeping the size of the study area fixed. This approach increases both the potential interaction distance between individuals and the habitat heterogeneity within local communities, key parameters for competition and habitat filtering, respectively (Weiher et al. 2011). At each grain size, we used the independent swap algorithm (Gotelli and 
Graves 1996) to generate a null distribution of 999 random communities for each observed community, using the local community $\times$ species matrix for that grain size. The independent swap null model provides a more conservative test of assembly processes by maintaining both observed species occurrence frequencies and local community species richness in the null model. Again, we used a Wilcoxon signed-rank test to assess, separately for each grain size, whether the distribution of the differences between the observed local community values at that grain size and their respective null model means was shifted away from zero. Because the signal of niche-based processes might be masked from MPD and MNTD analyses, owing to their assessment of pattern across the whole phylogeny, we also tested whether individual clades were filtering into specific habitat types. To achieve this, we grouped taxa by their primary habitat type (forest or nonforest; see app. A) and used the nodesig analysis in Phylocom (Webb et al. 2008) to test whether taxa descended from each node were overor underrepresented in a habitat type.

An absence of trait clustering within communities at larger spatial scales could result from an increase in habitat heterogeneity encompassed within these communities, as this could lead to a more dispersed functional trait set within communities. We used PCA to generate vegetation structure indexes from measured habitat variables and correlogram and mantel tests to quantify the degree of habitat (vegetation structure) heterogeneity across scales. We then used least squares regression to test whether there was a relationship at the smallest spatial scale between habitat type and the rank of a community's mean trait value in the null distribution (i.e., the deviation of community means from those expected under the null model). See appendix A for full details of vegetation surveys and spatial autocorrelation analysis.

\section{Results}

\section{Functional Traits}

Because standard PCA produced ambiguous niche axes (see app. A; table A3), we used a two-step PCA in which the first step was focused on describing candidate niche axes separating locomotory (wing, tail, and tarsus length) and trophic (beak length, width, and depth) traits (fig. A3; table A4). In all further analyses of community structure, we used the body-size, tarsus-to-tail-length-ratio, and beak-shape axes from the two-step PCA because they are realistic - as shown by the correlation with two axes from the standard PCA approach — and because they also provide an index of beak shape as an additional, more interpretable axis of the foraging niche. See appendix A for exact definitions, selection methods, and justification.
Community Assembly Simulations and Power Analyses: Multiple-Niche-Axis and Single-Niche-Axis Metrics

In the presence of a single community assembly process (either habitat filtering or competitive exclusion), standard trait-based multipattern, multiple-niche-axis metrics-FD and CHV - had high power to detect the correct assembly process (fig. 3). However, when both processes were occurring ( $75: 25$ or $50: 50), \mathrm{FD}$ and $\mathrm{CHV}$ had low or very low power to detect the dominant process or either process and detected competition over habitat filtering in the $50: 50$ scenario. An exception was that when the ratio of habitat filtering to competition was $25: 75, \mathrm{FD}$ and $\mathrm{CHV}$ had relatively high power to detect competition as the dominant process. Performance of FD and CHV was the same when the trait axis (body size) exposed to both habitat filtering and competition was switched with the trait axis (locomotory traits) exposed only to habitat filtering in the community simulation, except for the $50: 50$ case, where for competition, FD power increased and CHV power decreased (fig. A5). This suggests that the power of these metrics was relatively robust to differences between the trait axes in the initial distribution of species' trait values in the species pool. The multipattern, multipleniche-axis phylogenetic metrics-MPD and MNTDgenerally had even lower power to detect habitat filtering and competition than the trait-based metrics (figs. 3, A5).

In contrast, the trait-based multipattern, single-nicheaxis metrics-FD, variance, and range- had high power to detect the signature of competition at all levels of relative importance when used on a trait axis (beak shape) on which only competition was acting directly (figs. 4, A6). Variance and range also had high power to detect habitat filtering at all levels of its relative importance when it was the only process acting directly on body size (fig. 4). However, these metrics were not as successful in detecting the signature of habitat filtering when it was the only process acting directly on locomotory trait structure (fig. A6).

Multipattern, single-niche-axis metrics also improved on multipattern, multiple-niche-axis metrics by more often detecting the dominant niche-based process when multiple processes were active on a single-niche axis (body size; figs. A5, A6). However, the main difficulty for multipattern, single-niche-axis metrics also arose when there was the combination of habitat filtering and competition acting directly on the same niche axis, as under these scenarios, $\mathrm{FD}$, variance, and range were often unable to reject the random assembly null model (figs. 4, A6). In addition, FD and variance (for body size; fig. 4) and variance and range (for locomotory traits; fig. A6) detected regular spacing when competitive exclusion had not acted directly on the axis being tested but was the sole process active in community assembly (i.e., acting on other trait axes). 
The single-pattern, single-niche-axis metric, SDNDr, had low power to detect competition on beak shape, the axis on which only competition was acting directly, but had higher power to detect competition on the body-size axis (figs. 4, A6). However, SDNDr was also sensitive to habitat filtering on all three trait axes.

\section{Tests of Bird Community Assembly across Scales}

Multiple-Niche-Axis Tests. For trait-based metrics, we found no significant deviation from the random assembly null model expectation for bird community structure at any grain size of the local community for either FD or CHV (table 1; fig. 5). Thus, according to these metrics, community structure appeared to be purely stochastic. The only pattern that approached significance was of reduced volume (i.e., trait clustering) in observed $\mathrm{CHV}$ values at the 0.8 -ha scale (table 1 ).

When we tested the assumption of phylogenetic signal in traits, we found that species body size, beak shape (trophic trait), and tarsus to tail length ratio (locomotory trait) showed significantly more phylogenetic signal than expected if the distribution of trait values were random with respect to phylogeny but were less conserved than expected from a Brownian motion model of trait evolution (fig. 2; table A6). Phylogenetic signal in primary habitat type was not significantly different from that expected under either a random, nonphylogenetic model (estimated $D=0.65, P=.1$ ) or a Brownian motion model (estimated $D=0.65, P=.09)$. When we focused on community phylogenetic structure, we found that observed structure was not significantly different from the null model at any grain size for either MNTD or MPD (table 1). Some habitat filtering was evident, at least at the studysite scale, as the node at the base of the Synallaxis and Asthenes clade was significantly overrepresented in nonforest habitat (fig. 2). However, this was the only node significantly over- or underrepresented in any habitat type.
Single-Niche-Axis Tests. In contrast to multiple-niche-axis metrics, single-niche-axis approaches revealed complex nonrandom patterns of trait values within communities at smaller grain sizes (table 2; fig. 5). There were signals of both clustering and regular spacing in locomotory traits in communities at small spatial scales (0.8-1.6 ha) and the signal of regular spacing in body size and trophic traits (beak shape) at the smallest spatial scale $(0.8 \mathrm{ha})$. The multipattern, single-niche-axis metrics-variance and range-detected clustering in species trait values for tarsus to tail length ratio, with significantly reduced values for tarsus to tail length ratio in observed communities at the 0.8-ha scale. Beak shape and body size showed no significant reduction in variance or range at any spatial scale. The single-pattern, single-niche-axis metric, SDNDr, detected a pattern of regular spacing of species trait values for body size, tarsus to tail length ratio, and beak shape, as the observed community values for these traits were significantly shifted below the null model expectation within communities at the 0.8 -ha scale. At the 1.6-ha scale, only tarsus to tail length ratio showed significant regular spacing. No traits showed clustering or regular spacing at larger spatial scales.

Further Tests of Habitat Filtering. The first principal component (habitat PC1) from the habitat PCA explained 71\% of the variation in habitat data and was interpreted as an index of vegetation structure (table A7); increasing values are associated with more forested habitat, including increased maximum canopy height and tree cover and decreased grass and shrub cover. There was spatial autocorrelation in vegetation structure across 0.8 -ha survey sites (Mantel test: $r=0.23, P<.001, N=72,10,000$ permutations). However, this effect decayed to zero at distances greater than those separating neighboring 0.8-ha survey points (fig. A4), indicative of increased habitat heterogeneity for communities at larger spatial scales. At the 0.8-ha scale, there was a positive relationship between the vegetation structure (log habitat PC1) and the rank in the

Table 1: Results of multipattern, multiple-niche-axis trait and phylogenetic tests for habitat filtering and interspecific competition using functional diversity (FD), convex hull volume (CHV), mean phylogenetic distance (MPD), and mean nearest taxon distance (MNTD) at four nested spatial scales (grain sizes)

\begin{tabular}{|c|c|c|c|c|c|c|c|c|}
\hline \multirow[b]{2}{*}{ Spatial scale (ha) } & \multicolumn{4}{|c|}{ Trait-based metric } & \multicolumn{4}{|c|}{ Phylogeny-based metric } \\
\hline & FD & $P$ & $\mathrm{CHV}$ & $P$ & MPD & $P$ & MNTD & $P$ \\
\hline .8 & $.125 \pm .11$ & .26 & $.014 \pm .11$ & .06 & $.130 \pm .10$ & .38 & $.030 \pm .11$ & .91 \\
\hline 1.6 & $.002 \pm .15$ & .88 & $-.130 \pm .13$ & .16 & $.000 \pm .15$ & .80 & $-.140 \pm .17$ & .38 \\
\hline 3.2 & $.010 \pm .22$ & .96 & $-.175 \pm .20$ & .26 & $.050 \pm .20$ & .52 & $.050 \pm .42$ & .73 \\
\hline 6.4 & $.006 \pm .43$ & 1.00 & $.088 \pm .40$ & .36 & $.140 \pm .24$ & .43 & $.050 \pm .42$ & .91 \\
\hline
\end{tabular}

Note: Values are mean standard effect sizes (SES) \pm SE (SES calculated as observed value minus null model mean, divided by SD of the null model distribution, averaged across all observed communities at each spatial scale). Positive SES indicates even spacing; negative SES indicates clustering relative to the expected null model trait spacing or relatedness of the community. 


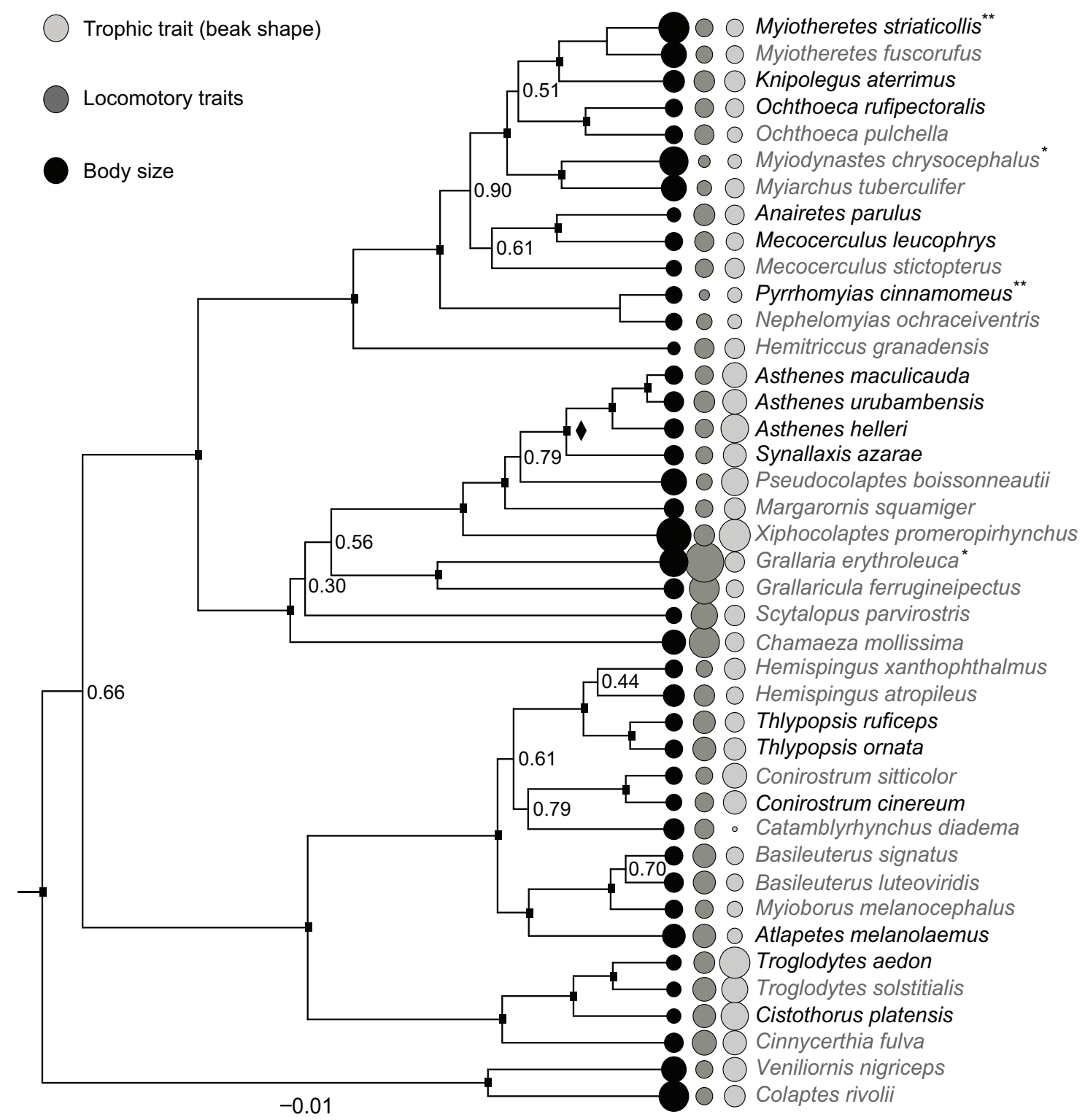

Figure 2: Maximum clade credibility tree inferred from analysis of mitochondrial DNA regions for all study species, labeled as occurring primarily in forest (gray names) or grassland and shrubby vegetation (black names). The diamond indicates the only node significantly overrepresented by a particular habitat (nonforest). The size of data circles represents the value of scaled traits: trophic traits (principal component [PC] 2; beak shape), locomotory traits (PC2; tarsus to tail length ratio), and body size (PC1). Nodes with squares indicate posterior probability (PP) support $>95 \%$; other PP values are given above nodes. One asterisk denotes species lacking genetic data but placed according to sequences from closely related substitute species (Megarynchus pitangua and Grallaria ruficapilla, respectively). Two asterisks denote species inserted on the basis of published phylogenies, with varying branch lengths (see "Methods"). Scale bar indicates sequence divergence. This maximum clade credibility tree, excluding the two species inserted based on published phylogenies, is available from TreeBASE: http://purl.org/phylo/treebase/phylows/study/TB2:S16064.

null distribution of community mean tarsus to tail length ratios $\left(\beta=1.94, r^{2}=0.25, P<.001, N=72\right)$. This shows that tarsus to tail length ratios were higher than expected by chance for species in forested sites and lower than expected for those in nonforest sites.

\section{Discussion}

\section{Disentangling Multiple Assembly Processes}

Our simulation analyses show that multiple-niche-axis phylogenetic and trait-based metrics have low power to detect 
Table 2: Results of multipattern, single-niche-axis and single-pattern, single-niche-axis trait metric tests for habitat filtering and interspecific competition across four nested spatial scales

\begin{tabular}{|c|c|c|c|c|c|c|c|c|}
\hline \multirow[b]{2}{*}{ Trait, spatial scale (ha) } & \multicolumn{2}{|l|}{ FD } & \multicolumn{2}{|c|}{ Variance } & \multicolumn{2}{|l|}{ Range } & \multicolumn{2}{|l|}{ SDNDr } \\
\hline & $\mathrm{SES} \pm \mathrm{SE}$ & $P$ & $\mathrm{SES} \pm \mathrm{SE}$ & $P$ & $\mathrm{SES} \pm \mathrm{SE}$ & $P$ & $\mathrm{SES} \pm \mathrm{SE}$ & $P$ \\
\hline \multicolumn{9}{|l|}{ Body size (PC1): } \\
\hline .8 & $-.050 \pm .10$ & .11 & $.032 \pm .13$ & .28 & $.042 \pm .12$ & .70 & $-.012 \pm .13$ & $<.05$ \\
\hline 1.6 & $-.111 \pm .20$ & .77 & $-.013 \pm .18$ & .96 & $-.024 \pm .17$ & .87 & $-.033 \pm .17$ & .48 \\
\hline 3.2 & $-.196 \pm .18$ & .10 & $-.011 \pm .24$ & .83 & $.114 \pm .23$ & .42 & $.124 \pm .21$ & .86 \\
\hline 6.4 & $-.127 \pm .31$ & 1.00 & $-.039 \pm .44$ & .82 & $-.147 \pm .44$ & .82 & $-.157 \pm .33$ & .46 \\
\hline \multicolumn{9}{|l|}{ Trophic traits (PC2): ${ }^{a}$} \\
\hline .8 & $-.142 \pm .15$ & .82 & $-.115 \pm .14$ & .78 & $-.166 \pm .14$ & .76 & $-.235 \pm .13$ & $<.05$ \\
\hline 1.6 & $-.077 \pm .22$ & .65 & $-.114 \pm .18$ & .88 & $-.079 \pm .19$ & .58 & $-.069 \pm .20$ & .15 \\
\hline 3.2 & $-.126 \pm .30$ & .47 & $-.059 \pm .30$ & .93 & $-.067 \pm .29$ & .93 & $-.120 \pm .25$ & .25 \\
\hline 6.4 & $-.094 \pm .28$ & .16 & $-.069 \pm .40$ & .65 & $-.087 \pm .29$ & .25 & $.004 \pm .31$ & .33 \\
\hline \multicolumn{9}{|c|}{ Locomotory traits (PC2): } \\
\hline .8 & $-.138 \pm .11$ & .28 & $-.085 \pm .10$ & $<.01$ & $-.123 \pm .10$ & $<.05$ & $-.191 \pm .08$ & $<.01$ \\
\hline 1.6 & $-.159 \pm .16$ & .17 & $-.065 \pm .15$ & .30 & $-.098 \pm .14$ & .46 & $-.144 \pm .13$ & $<.05$ \\
\hline 3.2 & $-.169 \pm .27$ & .58 & $-.034 \pm .23$ & .67 & $-.093 \pm .23$ & .67 & $-.196 \pm .17$ & .11 \\
\hline 6.4 & $-.034 \pm .41$ & .73 & $.043 \pm .33$ & .65 & $-.030 \pm .32$ & .65 & $-.171 \pm .25$ & .25 \\
\hline
\end{tabular}

Note: Values are mean standard effect sizes $(\mathrm{SES}) \pm$ SE (SES calculated as observed value minus null model mean, divided by SD of the null model distribution, averaged across all observed communities at each spatial scale). FD $=$ functional diversity; SDNDr $=$ standard deviation in neighbor distances divided by range. $P$ values are for Wilcoxon signed-rank tests.

a Beak shape.

b Tarsus to tail length ratio.

competition and habitat filtering when these processes act simultaneously, even when one process is dominant (fig. 3). We also show that, even when a combination of multipleniche-axis metrics is used, these methods can oversimplify assembly dynamics by detecting only a single niche-based process when multiple processes co-occur. This problem can arise when either subordinate processes are overlooked or multiple processes are equally influential, in both cases exaggerating the impression that assembly processes occur in isolation rather than in combination.

These findings cast doubt on numerous trait-based analyses using multiple-niche-axis metrics to detect processes acting simultaneously. The multiple-niche-axis approach is often supported with reference to previous simulation studies, several of which have shown FD, CHV, and abundance-weighted multiple-niche-axis trait metrics (e.g., Rao's quadratic entropy) to be powerful metrics for detecting niche-based processes. However, these studies either (1) evaluated trait metrics when only limiting similarity or habitat filtering was present (e.g., Mouchet et al. 2010), (2) focused niche-based processes on generating only trait clustering within local communities (Münkemüller et al. 2012), (3) focused community assembly on a single trait axis (Mason et al. 2013), or (4) applied assembly processes equally across all trait axes (Mouchet et al. 2010; Münkemüller et al. 2012; Aiba et al. 2013). Therefore, previous studies do not address many of the cases in community assembly where multiple processes differ in their relative importance across multiple niche axes (e.g., Spasojevic and Suding 2012), as seems likely when testing for assembly processes across spatial scales.

When we applied multiple-niche-axis-trait (FD and CHV) and phylogenetic (MPD and MNTD) metrics to our observed study communities, none of them detected significant deviations from the random assembly null model expectations (table 1; fig. 5). Although the absence of any phylogenetic or trait clustering may be relatively uninformative, as the influence of habitat filtering is often assumed to be weak at these smaller scales (0.8-6.4 ha), the lack of evidence for overdispersion of functional traits or phylogenetic relationships is surprising, given that species interactions are expected to strengthen as spatial scale decreases (Swenson et al. 2007; Cavender-Bares et al. 2009). Nonetheless, an absence of both clustering and overdispersion in traits and phylogenies has been reported fairly widely in plants and for some animal communities at local scales and is typically interpreted as evidence for a neutral model of community assembly (e.g., Gómez et al. 2010; Thompson et al. 2010).

A strongly contrasting set of patterns was detected by single-niche-axis metrics (FD, variance, range, and SDNDr). Simulations revealed that these metrics are able to detect the action of both competition and habitat filtering across a wide range in the relative importance of either process (fig. 4). In accordance, single-niche-axis metrics also identified significant clustering and regular spacing of traits in 


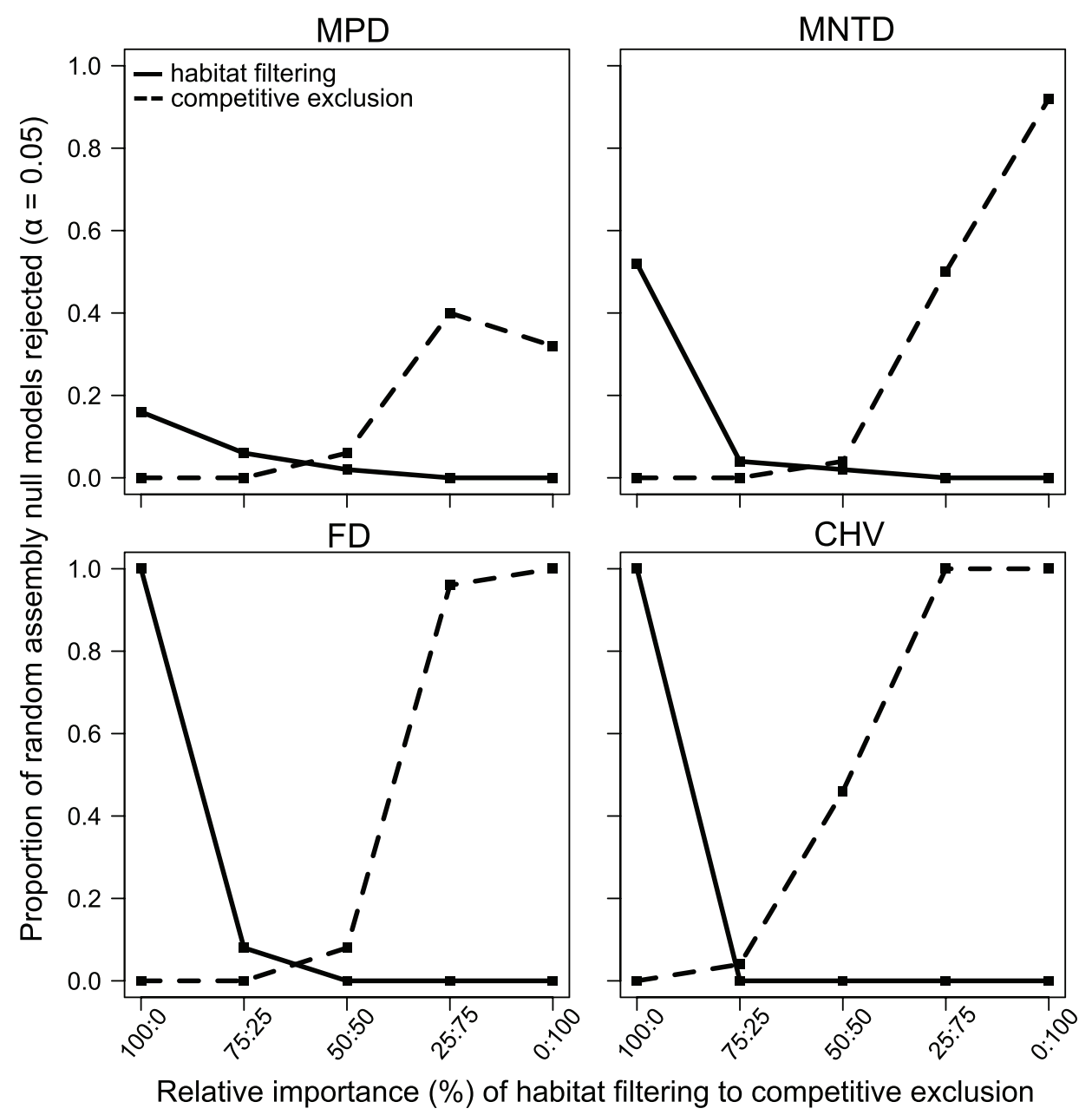

Figure 3: Multiple-niche-axis metrics: the power of multipattern, phylogenetic, and functional trait metrics-mean phylogenetic distance (MPD), mean nearest taxon distance (MNTD), functional diversity (FD), and convex hull volume (CHV) - to reject the random community assembly null model in favor of either habitat filtering or competitive exclusion. Assembly models were tested under five scenarios ranging in the relative importance of habitat filtering and competitive exclusion from the sole action of one process to a 50:50 mixing of both processes. Each metric was calculated using multiple niche axes, and each species is a composite of three traits: body size, locomotory traits, and beak traits. During community assembly simulations, body size was subjected directly to habitat filtering and competitive exclusion, locomotory traits to habitat filtering only, and beak shape to competitive exclusion only. See "Methods" for further details of trait and community simulations, null models, and statistical tests.

observed communities (table 2; fig. 5). These nonrandom patterns reject neutral assembly models and suggest instead that habitat filtering and interspecific competition act simultaneously on locomotory traits, while interspecific competition acts on body size and beak shape, to structure bird communities at small grain sizes (0.8-1.6 ha).

Taken together, these results confirm previous suggestions that multiple-niche-axis metrics-because they measure the net effect of different assembly processes-may be ineffective at differentiating niche-based processes from neutral dynamics because multiple, opposing niche-based processes can cancel each other out (Kraft et al. 2007;
Swenson and Enquist 2009; Weiher et al. 2011). Moreover, we have shown that this is often the case even when either habitat filtering or interspecific competition plays a dominant role in community assembly. Finally, multiple-nicheaxis metrics generally detected only a single niche-based process, even when two processes were equally prevalent. Thus, multiniche-axis metrics may generate misleading evidence of either neutrality or the partitioning of assembly processes across scales. In contrast, because they do not integrate processes operating on separate niche axes, single-niche-axis metrics are less likely to combine the signal of multiple processes and thus more likely to detect the 

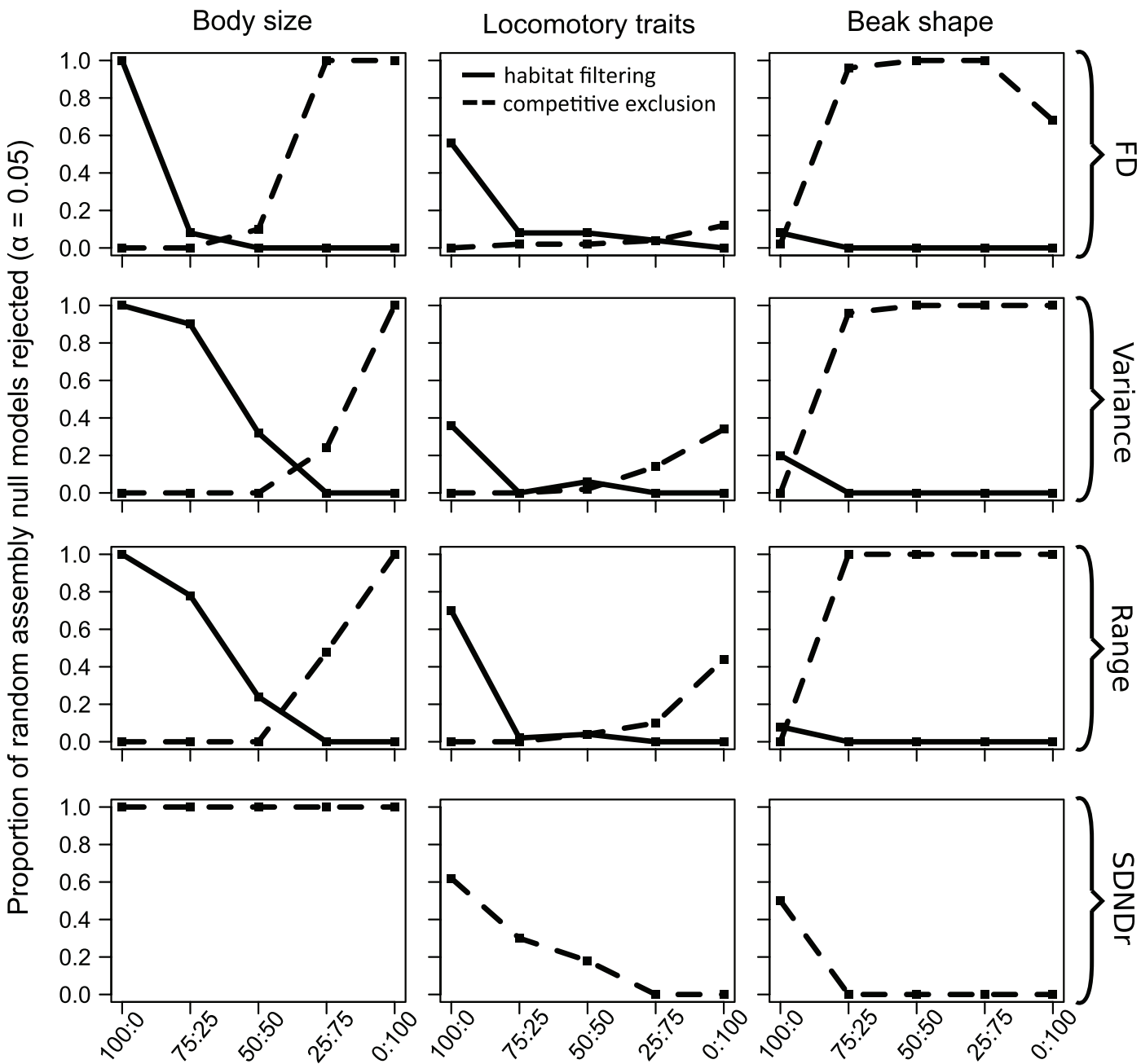

Relative importance (\%) of habitat filtering to competitive exclusion

Figure 4: Single-niche-axis metrics: the power of multipattern and single-pattern trait metrics, calculated using only a single-niche axis, to reject the random assembly null model in favor of either habitat filtering or competitive exclusion. Assembly models were tested under five scenarios ranging in the relative importance of habitat filtering and competitive exclusion from the sole action of one process to a $50: 50$ mixing of both processes. Functional diversity (FD) and standard deviation in neighbor distances are divided by range (SDNDr). Each species is a composite of three traits: body size, locomotory traits, and beak traits. During community assembly, the locomotory trait axis was subjected directly to habitat filtering and competitive exclusion, body size to habitat filtering only, and beak shape to competitive exclusion only. A single-pattern metric, SDNDr is focused on only the detection of competitive exclusion, and so a one-tailed test was used. See "Methods" for further details of trait and community simulations, null models, and statistical tests.

full set of processes present in community assembly at a given scale.

\section{Implications for Tests of Community Assembly}

Our findings highlight problems with multiple-niche-axistrait and phylogenetic analytical approaches but do not imply that such approaches should be abandoned. Multiaxis metrics, as confirmed by our simulations, can in some cases provide an integrated assessment of whether any particular niche-based assembly process is dominant (e.g., Petchey et al. 2007). They also make fewer assumptions about which trait axes are relevant to community assembly and can provide links between community assembly and ecosystem function (Cadotte et al. 2009; Thompson et al. 2010; but see Butterfield and Suding 2013), while phylogenetic approaches allow for analyses to be conducted when potentially important traits are difficult to measure (Cavender-Bares et al. 2009). Given these advantages, an important target for future research is to 

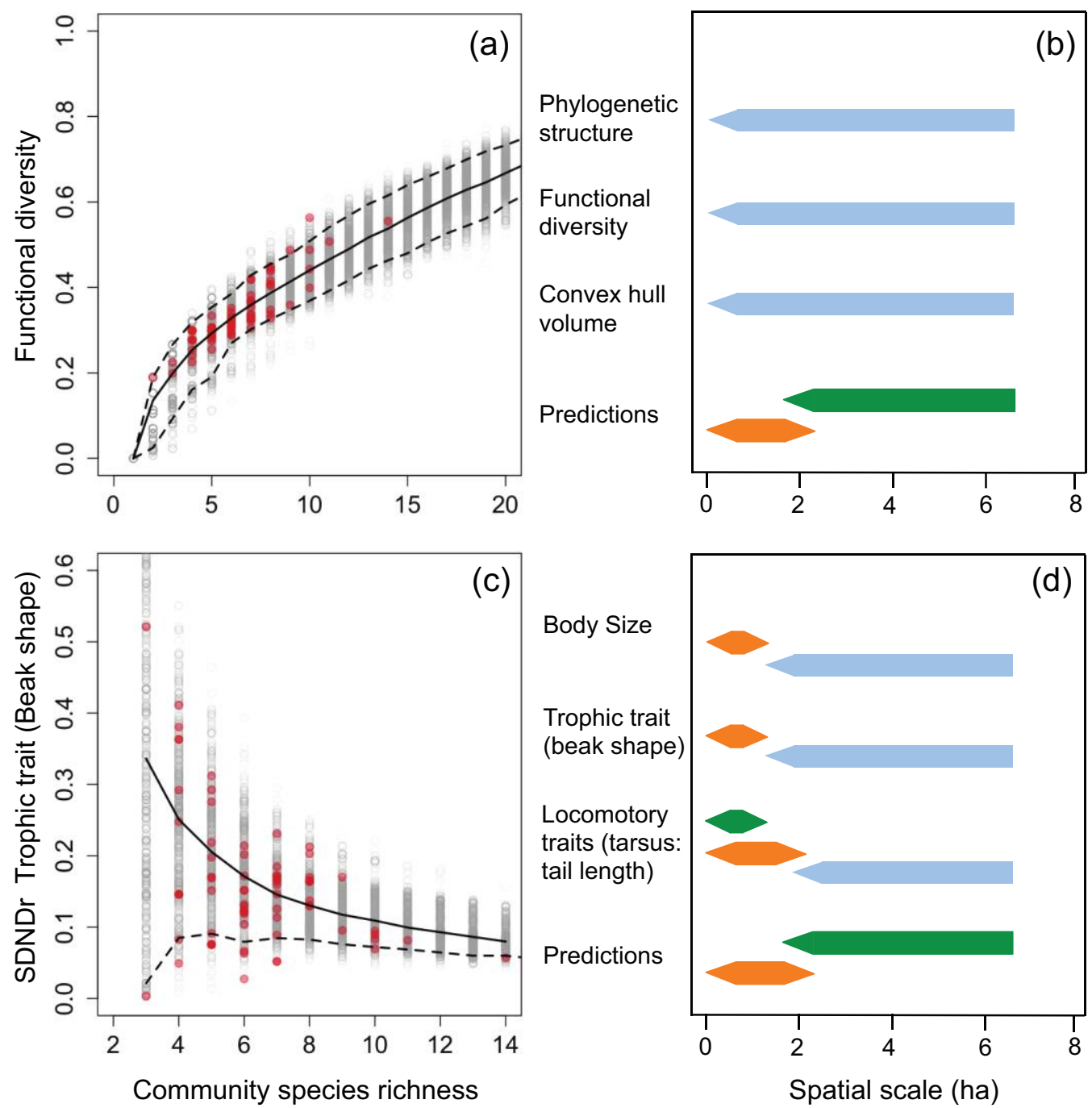

Clustering
(habitat filtering)

Regular spacing (competition)

Random pattern (null model expectation)

Figure 5: $a$, Observed community functional diversity values (red) do not differ significantly from the distribution of null community values (gray) at the 0.8-ha scale; mean (solid line) and 95\% confidence interval (dashed lines). $b$, No multipattern, multiple-niche-axis phylogenetic or trait-based test rejects the null model at any spatial scales (grain sizes 0.8-6.4 ha). c, The distribution of a single-pattern, single-niche-axis metric (SDNDr) is significantly shifted below the null expectation mean, suggesting that beak shapes are more regularly spaced than expected under random assembly at the 0.8 -ha scale. Only one interval is indicated because the test is one-tailed. $d$, Testing individual trait axes using single-niche-axis metrics yielded significant deviation from the null expectation, consistent with habitat filtering and competition structuring communities at smaller spatial scales (grain sizes 0.8-6.4 ha).

investigate whether the sensitivity of such multipattern, multiple-niche-axis metrics can be increased, for example, by systematically adjusting the weighting of a trait to test for its influence on the overall trait pattern and developing metrics more targeted to detecting a single niche-based process.

Single-niche-axis metrics, because they do not integrate processes operating on separate niche axes, aid interpre- tation of the action of niche-based processes on specific niche axes, thus enabling a more detailed description of how niche-based processes influence community assembly. An inherent challenge of single-niche-axis methods is to approximate niche axes from trait data, and we have shown that this is not always achievable using the classic approach of a PCA, as this may combine information from multiple niche axes on a single principal component axis. As an 
alternative, we introduce a technique of performing separate PCAs on groups of traits according to their acknowledged relevance to particular niche axes (see app. A; fig. A3). The main advantage of this approach is that specific tests of assembly processes can be focused exclusively on independent niche dimensions identified by a priori hypotheses. For example, species interactions can be tested on trophic traits separately from locomotory traits, as some of the latter are not expected to mediate interspecific competition and indeed may blur the signal of competitive exclusion.

Our results indicate that single-niche-axis metrics can struggle in two different scenarios. First, the power of multipattern metrics is reduced when multiple processes act on a single-niche axis. Second, both multi- and singlepattern, single-niche-axis metrics can sometimes wrongly detect the presence of a process when that process is absent on the trait axis being tested but strongly prevalent on another axis, even when the axes are uncorrelated (fig. 4). Similarly, the lower power of SDNDr to detect regular spacing in our trophic trait (beak shape) suggests that some single-pattern metrics may have lower power for trait axes with lower variance, where perhaps assembly patterns are weaker than on axes with higher variance. Thus, we recommend the complementary use of both multipattern and single-pattern metrics focused on single-niche axes, as this can enable detection of insensitivity or error in the metrics. We discourage the use of multipattern metrics such as variance and range, which are sensitive to both clustering and overdispersion, in single-pattern, one-tailed hypothesis tests, as has been advocated previously (Kraft and Ackerly 2010; Aiba et al. 2013). We have also shown that SDNDr, a single-pattern metric for competition, is sensitive to habitat filtering and therefore should be interpreted cautiously if other metrics detect clustering on the same axis. Further research is required to develop metrics that are sensitive to only one of either clustering or overdispersion and that are also robust to differences in variance among traits.

The study of community assembly processes has advanced rapidly in recent decades, driven in part by the development of sophisticated analytical techniques (Cavender-Bares et al. 2009). In general, the tendency has been to develop methods for pooling together as much information as possible, with recent studies even advocating the combined analysis of multiple trait and phylogenetic axes (Cadotte et al. 2013). While such all-inclusive approaches have a number of advantages, our analyses indicate that they can obscure the nuances of community assembly over time and space. This finding supports a recent countertrend in community ecology that proposes not the integration of data sets but their subdivision into niche axes before analysis (e.g., Spasojevic and Suding
2012). We suggest that studies of community assembly need to consider both inclusive and more targeted approaches to tease apart the influence of multiple assembly processes on community structure.

\section{Implications for Bird Community Assembly}

The regular spacing detected by single-niche-axis metrics in functional traits associated with locomotion and foraging is consistent with interspecific competition at the 0.8-1.6-ha scale and implies that bird communities at these grain sizes are structured by interspecific interactions such as competitive exclusion through direct interspecific territory defense or indirect competition for resources. Although the evidence we present aligns closely with longstanding ideas about the importance of beak differences (Hutchinson 1959; Schoener 1965) and foraging microhabitat divergence (MacArthur 1958; MacArthur and Levins 1967) in promoting co-occurrence, it suggests that the spatial scale of these interactions can be highly localized. This result contradicts recent suggestions that for mobile species such as birds that are not forced to interact as intimately with their neighbors as sessile organisms, the importance of niche processes may be low at smaller local scales (Weiher et al. 2011; Harmon-Threatt and Ackerly 2013). One possible reason for the absence of a signal of interspecific competition at larger grain sizes is that the size of individual territories for many of our study species is likely to be only 1-2 ha (Kikuchi 2009), meaning that competing species can potentially co-occur in larger communities without coming into direct contact, thus weakening the signal of competitive exclusion at increasing grain sizes. The reduced likelihood of direct competition at larger grain sizes may also explain why Gómez et al. (2010) found no evidence of competition structuring Neotropical antbird communities in plots of $100 \mathrm{ha}$, as the mean territory size of their study species is only $\sim 5$ ha (Terborgh et al. 1990). Also, by focusing on a single family, as opposed to on all insectivorous bird species in a community, Gómez et al. (2010) may have excluded important interspecific competitive interactions.

Single-niche-axis metrics revealed not only regular spacing but also clustering in locomotory traits, suggesting habitat filtering among communities at small grain sizes (0.8 ha). The positive relationship we detected between the vegetation structure and the mean locomotory trait (tarsus to tail length ratio) values of 0.8 -ha communities suggests that habitat filtering is related to traits associated with foraging maneuver and substrate use and, specifically, the consistent differences between traits in forest versus nonforest sites. This relationship between habitat type and community mean tarsus to tail length ratio also suggests that trait clustering is likely not due to a competitive hi- 
erarchy in these locomotory traits (Mayfield and Levine 2010; HilleRisLambers et al. 2012). We found no evidence of trait clustering above a grain size of 0.8 ha. This is likely because local assemblages of increased grain size (1.6-6.4 ha) encompassed greater habitat heterogeneity (both forest and nonforest vegetations) and thus contained sets of species with both higher and lower than expected tarsus to tail length ratios, weakening the signal of habitat filtering.

Community ecology studies generally predict that habitat filtering will be a dominant influence on community assembly at larger grain sizes than interspecific competition because habitat features vary over distances larger than those over which individuals interact (Swenson et al. 2007; Kraft and Ackerly 2010; Smith et al. 2013), but most previous studies have focused on sessile taxa (Vamosi et al. 2009). We found that this relationship was reversed in our study, with both habitat filtering and interspecific competition acting at small grain sizes and the grain size of interspecific competition ( $1.6 \mathrm{ha}$ ) extending beyond that of habitat filtering ( $0.8 \mathrm{ha})$. We suggest that across small to intermediate spatial extents, this pattern may be relatively common in mobile taxa that are able to interact through either direct interspecific territory defense or indirect competition for resources across the same or greater distances than variation in habitat features.

We have interpreted the regular spacing of functional traits as evidence of competitive exclusion and clustering as evidence of habitat filtering, following many previous studies. Other processes may, however, contribute to these patterns. For example, social information gathering could influence spacing between conspecifics and even heterospecifics, potentially shaping community structure (Seppänen et al. 2007). This process seems unlikely to lead to pervasive overdispersion or even clustering in functional traits at the scales we detect, particularly as most of our study lineages hold year-round territories. In addition, intraspecific variation could affect community dynamics by altering species' niche widths (Bolnick et al. 2011). Although this may lead to changes in patterns of niche overlap, the majority of trait variation was among species and not individuals (table A2), and we suspect that intraspecific variation is unlikely to drive the patterns we have detected.

Our results add to a growing body of work seeking to understand the role of deterministic and stochastic processes across scales (Levin 1992; Chase and Myers 2011; Chave 2013). The patterns we detect are consistent with the idea that niche-based processes minimize spatial overlaps between species sharing similar ecologies, particularly in tropical systems where the ranges of ecological competitors often abut (Terborgh and Weske 1975; Jankowski et al. 2010; Pigot and Tobias 2013). Further research is needed to determine whether similar processes structure avian communities in temperate systems where many spe- cies hold territories only during the breeding season, potentially altering the intensity and spatial scale of competition (Gotelli et al. 2010).

\section{Acknowledgments}

We thank P. Birget, R. Butron, M. Cabrera, B. Daly, G. Jamie, M. Jurado, M. Livrand, and C. Santos for assistance with fieldwork; the Amazon Conservation Association (ACA) and the Centro de Ornitología y Biodiversidad (CORBIDI) for logistical assistance in Peru; Servicio Nacional de Áreas Naturales Protegidas (SERNANP) for granting research permission; and the Natural History $\mathrm{Mu}-$ seum at Tring and the Oxford University Natural History Museum for access to specimens. We are also grateful to N. Kraft, A. Pigot, N. Seddon, and C. Webb for useful comments or discussion. Three anonymous reviewers and B. McGill provided comments that significantly improved this work. This research was funded by a Rhodes Scholarship (C.H.T.) and a grant from the John Fell Fund (J.A.T).

\section{Literature Cited}

Aiba, M., M. Katabuchi, H. Takafumi, S. S. Matsuzaki, T. Sasaki, and T. Hiura. 2013. Robustness of trait distribution metrics for community assembly studies under the uncertainties of assembly processes. Ecology 94:2873-2885.

Blomberg, S. P., T. Garland, and A. R. Ives. 2003. Testing for phylogenetic signal in comparative data: behavioral traits are more labile. Evolution 57:717-745.

Bolnick, D. I., P. Amarasekare, M. S. Araújo, R. Bürger, J. M. Levine, M. Novak, V. H. W. Rudolf, et al. 2011. Why intraspecific trait variation matters in community ecology. Trends in Ecology and Evolution 26:183-192.

Butterfield, B. J., and K. N. Suding. 2013. Single-trait functional indices outperform multi-trait indices in linking environmental gradients and ecosystem services in a complex landscape. Journal of Ecology 101:9-17.

Cadotte, M. W. 2006. Metacommunity influences on community richness at multiple spatial scales: a microcosm experiment. Ecology 87:1008-1016.

Cadotte, M. W., C. H. Albert, and S. C. Walker. 2013. The ecology of differences: assessing community assembly with trait and evolutionary distances. Ecology Letters 16:1234-1244. doi:10.1111 /ele.12161.

Cadotte, M. W., J. Cavender-Bares, D. Tilman, and T. H. Oakley. 2009. Using phylogenetic, functional and trait diversity to understand patterns of plant community productivity. PLoS ONE 4: e5695.

Cavender-Bares, J., A. Keen, and B. Miles. 2006. Phylogenetic structure of Floridian plant communities depends on taxonomic and spatial scale. Ecology 87:109-122.

Cavender-Bares, J., K. H. Kozak, P. V. A. Fine, and S. W. Kembel. 
2009. The merging of community ecology and phylogenetic biology. Ecology Letters 12:693-715.

Chao, A. 1987. Estimating the population size for capture-recapture data with unequal catchability. Biometrics 43:783-791.

Chase, J. M., and J. A. Myers. 2011. Disentangling the importance of ecological niches from stochastic processes across scales. Philosophical Transactions of the Royal Society B: Biological Sciences 366:2351-2363.

Chave, J. 2013. The problem of pattern and scale in ecology: what have we learned in 20 years? Ecology Letters 16(suppl.):4-16.

Colwell, R. K. 2000. EstimateS: statistical estimation of species richness and shared species from samples (software and user's guide). Version 8.2.0. http://viceroy.eeb.uconn.edu/estimates/.

Cornwell, W. K., L. D. W. Schwilk, and D. D. Ackerly. 2006. A traitbased test for habitat filtering: convex hull volume. Ecology 87: $1465-1471$.

Fritz, S. A., and A. Purvis. 2012. Selectivity in mammalian extinction risk and threat types: a new measure of phylogenetic signal strength in binary traits. Conservation Biology 24:1042-1051.

Gause, G. F. 1934. The struggle for existence. Williams \& Wilkins, Baltimore.

Gibbon, A., M. R. Silman, Y. Malhi, J. B. Fisher, P. Meir, M. Zimmermann, G. C. Dargie, W. R. Farfan, and K. C. Garcia. 2010 Ecosystem carbon storage across the grassland-forest transition in the high Andes of Manu National Park, Peru. Ecosystems 13: 1097-1111.

Gómez, J. P., G. A. Bravo, R. T. Brumfield, J. G. Tello, and C. D. Cadena. 2010. A phylogenetic approach to disentangling the role of competition and habitat filtering in community assembly of Neotropical forest birds. Journal of Animal Ecology 79:1181-1192.

Gotelli, N. J., and G. R. Graves. 1996. Null models in ecology. Smithsonian Institution, Washington, DC.

Gotelli, N. J., G. R. Graves, and C. Rahbek. 2010. Macroecological signals of species interactions in the Danish avifauna. Proceedings of the National Academy of Sciences of the USA 107:5030-5035.

Grant, P. R., and B. R. Grant. 2006. Evolution of character displacement in Darwin's finches. Science 313:224-226.

Harmon-Threatt, A. N., and D. D. Ackerly. 2013. Filtering across spatial scales: phylogeny, biogeography and community structure in bumble bees. PLoS ONE 8:e60446.

HilleRisLambers, J., P. B. Adler, W. S. Harpole, J. M. Levine, and M. M. Mayfield. 2012. Rethinking community assembly through the lens of coexistence theory. Annual Review of Ecology, Evolution, and Systematics 43:227-248.

Hubbell, S. P. 2001. The unified neutral theory of biodiversity and biogeography. Princeton University Press, Princeton, NJ.

Huston, M. A. 1999. Local processes and regional patterns: appropriate scales for understanding variation in the diversity of plants and animals. Oikos 86:393-401.

Hutchinson, G. E. 1959. Homage to Santa Rosalia, or why are there so many kinds of animals? American Naturalist 93:145-159.

Jankowski, J. E., S. K. Robinson, and D. J. Levey. 2010. Squeezed at the top: interspecific aggression may constrain elevational ranges in tropical birds. Ecology 91:1877-1884.

Kikuchi, D. W. 2009. Terrestrial and understorey insectivorous birds of a Peruvian cloud forest: species richness, abundance, density, territory size and biomass. Journal of Tropical Ecology 25:523529.

Kraft, N. J. B., and D. D. Ackerly. 2010. Functional trait and phy- logenetic tests of community assembly across spatial scales in an Amazonian forest. Ecological Monographs 80:401-422.

Kraft, N. J. B., W. K. Cornwell, C. O. Webb, and D. D. Ackerly. 2007. Trait evolution, community assembly, and the phylogenetic structure of ecological communities. American Naturalist 170:271-283.

Kraft, N. J. B., R. Valencia, and D. D. Ackerly. 2008. Functional traits and niche-based tree community assembly in an Amazonian forest. Science 322:580-582.

Levin, S. A. 1992. The problem of pattern and scale in ecology. Ecology 73:1943-1967.

Lovette, I. J., and W. M. Hochachka. 2006. Simultaneous effects of phylogenetic niche conservatism and competition on avian community structure. Ecology 87:14-28.

MacArthur, R., and R. Levins. 1967. The limiting similarity, convergence, and divergence of coexisting species. American Naturalist 101:377-385.

MacArthur, R. H. 1958. Population ecology of some warblers of northeastern coniferous forests. Ecology 39:599-619.

Mason, N. W., F. Bello, D. Mouillot, S. Pavoine, and S. Dray. 2013. A guide for using functional diversity indices to reveal changes in assembly processes along ecological gradients. Journal of Vegetation Science 24:794-806.

Mayfield, M. M., and J. M. Levine. 2010. Opposing effects of competitive exclusion on the phylogenetic structure of communities. Ecology Letters 13:1085-1093.

McGill, B. J. 2010. Ecology: matters of scale. Science 328:575-576.

Miles, D. B., and R. E. Ricklefs. 1984. The correlation between ecology and morphology in deciduous forest passerine birds. Ecology 65: 1629-1640.

Mouchet, M. A., S. Villéger, N. W. H. Mason, and D. Mouillot. 2010. Functional diversity measures: an overview of their redundancy and their ability to discriminate community assembly rules. Functional Ecology 24:867-876.

Münkemüller, T., F. De Bello, C. N. Meynard, D. Gravel, S. Lavergne, D. Mouillot, N. Mouquet, and W. Thuiller. 2012. From diversity indices to community assembly processes: a test with simulated data. Ecography 35:468-480.

Petchey, O. L., K. L. Evans, I. S. Fishburn, and K. J. Gaston. 2007. Low functional diversity and no redundancy in British avian assemblages. Journal of Animal Ecology 76:977-985.

Petchey, O. L., and K. J. Gaston. 2002. Functional diversity (FD), species richness and community composition. Ecology Letters 5: 402-411.

Pigot, A. L., and J. A. Tobias. 2013. Species interactions constrain geographic range expansion over evolutionary time. Ecology Letters $16: 330-338$.

R Core Team. 2013. R: a language and environment for statistical computing. R Foundation for Statistical Computing, Vienna.

Ricklefs, R. E., and J. Travis. 1980. A morphological approach to the study of avian community organization. Auk 97:321-338.

Robinson, S. K., and J. Terborgh. 1995. Interspecific aggression and habitat selection by Amazonian birds. Journal of Animal Ecology 64:1-11.

Schoener, T. W. 1965. The evolution of bill size differences among sympatric congeneric species of birds. Evolution 19:189-213.

Seppänen, J.-T., J. T. Forsman, M. Mönkkönen, and R. L. Thomson. 2007. Social information use is a process across time, space, and ecology, reaching heterospecifics. Ecology 88:1622-1633.

Smith, A. B., B. Sandel, N. J. B. Kraft, and S. Carey. 2013. Characterizing 
scale-dependent community assembly using the functional-diversityarea relationship. Ecology 94:2392-2402.

Spasojevic, M. J., and K. N. Suding. 2012. Inferring community assembly mechanisms from functional diversity patterns: the importance of multiple assembly processes. Journal of Ecology 100: 652-661.

Stubbs, W. J., and J. Wilson. 2004. Evidence for limiting similarity in a sand dune community. Journal of Ecology 92:557-567.

Swenson, N. G., and B. J. Enquist. 2009. Opposing assembly mechanisms in a Neotropical dry forest: implications for phylogenetic and functional community ecology. Ecology 90:2161-2170.

Swenson, N. G., B. J. Enquist, J. Thompson, and J. K. Zimmerman. 2007. The influence of spatial and size scale on phylogenetic relatedness in tropical forest communities. Ecology 88:1770-1780.

Terborgh, J., S. K. Robinson, T. A. Parker, C. A. Munn, and N. Pierpont. 1990. Structure and organization of an Amazonian forest bird community. Ecological Monographs 60:213-238.

Terborgh, J., and J. S. Weske. 1975. Role of competition in distribution of Andean birds. Ecology 56:562-576.

Thompson, K., O. L. Petchey, A. P. Askew, N. P. Dunnett, A. P. Beckerman, and A. J. Willis. 2010. Little evidence for limiting similarity in a long-term study of a roadside plant community. Journal of Ecology 98:480-487.

Trisos, C. H., O. L. Petchey, and J. A. Tobias. 2014. Data from: Unraveling the interplay of community assembly processes acting on multiple niche axes across spatial scales. American Naturalist, Dryad Digital Repository, http://doi.org/10.5061/dryad.k9m20.
Vamosi, S. M., S. B. Heard, J. C. Vamosi, and C. O. Webb. 2009. Emerging patterns in the comparative analysis of phylogenetic community structure. Molecular Ecology 18:572-592.

Vellend, M. 2010. Conceptual synthesis in community ecology. Quarterly Review of Biology 85:183-206.

Villéger, S., N. W. H. Mason, and D. Mouillot. 2008. New multidimensional functional diversity indices for a multifaceted framework in functional ecology. Ecology 89:2290-2301.

Violle, C., M. L. Navas, D. Vile, E. Kazakou, C. Fortunel, I. Hummel, and E. Garnier. 2007. Let the concept of the trait be functional. Oikos 117:1351-1361.

Webb, C. O., D. D. Ackerly, and S. W. Kembel. 2008. Phylocom: software for the analysis of phylogenetic community structure and trait evolution. Bioinformatics 24:2098-2100.

Webb, C. O., D. D. Ackerly, M. A. McPeek, and M. J. Donoghue. 2002. Phylogenies and community ecology. Annual Review of Ecology and Systematics 33:475-505.

Weiher, E., D. Freund, T. Bunton, A. Stefanski, T. Lee, and S. Bentivenga. 2011. Advances, challenges and a developing synthesis of ecological community assembly theory. Philosophical Transactions of the Royal Society B: Biological Sciences 366:2403-2413.

Weiher, E., and P. A. Keddy. 1995. Assembly rules, null models, and trait dispersion: new questions from old patterns. Oikos 74:159164.

Associate Editor: Brian McGill Editor: Troy Day

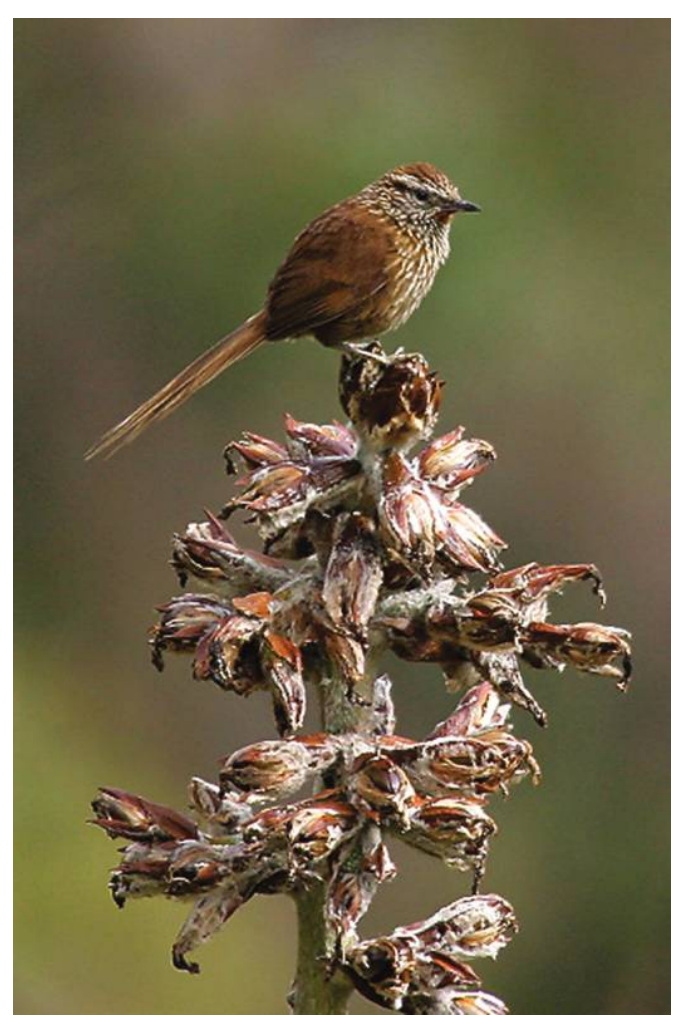

A line-fronted canastero (Asthenes urubambensis) in Tokilane, Bolivia. Photo credit: J. A. Tobias. 